\title{
Comparison of Visible, Thermal Infra-Red and Range Images for Face Recognition
}

\author{
Ajmal Mian \\ School of Computer Science and Software Engineering \\ The University of Western Australia \\ 35 Stirling Highway, Crawley, WA 6009, Australia \\ ajmal@csse.uwa.edu. au
}

\begin{abstract}
Existing literature compares various biometric modalities of the face for human identification. The common criterion used for comparison is the recognition rate of different face modalities using the same recognition algorithms. Such comparisons are not completely unbiased as the same recognition algorithm or features may not be suitable for every modality of the face. Moreover, an important aspect which is overlooked in these comparisons is the amount of variation present in each modality which will ultimately effect the database size each modality can handle. This paper presents such a comparison between the most common biometric modalities of the face namely visible, thermal infrared and range images. Experiments are performed on the Equinox and the FRGC databases with results indicating that visible images capture more interpersonal variations of the human face compared to thermal IR and range images. We conclude that under controlled conditions, visible face images have a greater potential of accommodating large databases compared to long-wave IR and range images.
\end{abstract}

\section{Introduction}

Face recognition is an important and challenging computer vision problem. It has many potential applications in security, surveillance and access control. One of the main challenges in face recognition is the fact that intra-class variations caused by changes in illumination, pose and facial expressions sometimes exceed inter-class variations. For example, different people tend to appear more similar from the same pose compared to the same person viewed from different poses. Likewise, different people in the same illumination condition sometimes appear more similar than the same person viewed in different illumination conditions. This is one of the main reasons why simple algorithms like Principal Component Analysis (PCA) 1] do not perform well under changing illumination, pose and facial expressions. A comprehensive survey of face recognition algorithms is given by Zhao et al. 2].

One way of dealing with the above challenges is to train the recognition system with multiple instances of each face under different conditions of illumination, expressions and pose. The training data are projected to a space which minimizes

T. Wada, F. Huang, and S. Lin (Eds.): PSIVT 2009, LNCS 5414, pp. 807-816, 2009.

(C) Springer-Verlag Berlin Heidelberg 2009 
the intra-class variation while maximizing the inter-class variation. This is the basic idea behind LDA (Linear Discriminant Analysis) based face recognition 3. One assumption in LDA is that the data are linearly separable which is not always true about faces. Quadratic Discriminant Analysis (QDA) [4] relaxes this assumption and defines a quadratic surface to separate the classes. However, a common problem in LDA and QDA is that sufficient training samples covering all possible illuminations, expressions and poses of every individual are not always available. This is known as the small sample size problem [5].

In a hope to find invariant facial biometrics, researchers have investigated biometric modalities of the face other than the visible spectrum images. These modalities include the appearance of the face in the infra-red (IR) spectrum and the geometric shape of the face represented as range images or 3D polygonal models. 3D models of the face are completely invariant to illumination as they represent the facial geometry rather than the reflective properties of the face which are a function of the incident light, the facial pose, the face albedo and the facial geometry. However, the acquisition of $3 \mathrm{D}$ faces is not a completely illumination invariant process as it relies on controlled active illumination of the face (e.g. with laser stripes) in order to triangulate the facial geometry [6]. On the positive side, once the $3 \mathrm{D}$ face is acquired along with its coregistered texture (visible image), an infinite number of training samples (visible images) under different illuminations and poses can be synthesized to overcome the small sample size problem. Blanz and Vetter [7] fitted a 3D morphable model to single face images and generated many training samples to represent the same face in different illuminations and poses. 3D face models can also be used to handle expression variations. For example, Bronstein et al. [8] used isometric deformations on textured 3D faces to alleviate the effects of expressions on the face.

The infra-red spectrum can be roughly divided into four different bandwidths namely, Near-IR (NIR), Short-wave-IR (SWIR), Medium-wave-IR (MWIR) and Long-wave-IR (referred to as thermal IR in this paper). Out of the four, only the first and the last modalities have been investigated in existing literature. Two arguments are generally presented in favor of NIR images. The first one is that off-the-shelf CCD sensors are sensitive to this bandwidth and normal cameras can be modified to acquire NIR images. The second argument is that NIR is not visible to the human eye and active controlled NIR illumination can be used to acquire facial images while the system is still imperceptible to humans 9 . It is not surprising that by controlling illumination conditions good face recognition results can be achieved. However, this still does not solve the pose problem as the appearance of NIR images of the same face will change with pose.

It would not be incorrect to say that thermal IR is truly invariant to illumination conditions. Unlike 3D face and NIR face images, thermal IR images do not require the active controlled illumination of faces. In fact, thermal IR can be captured in complete darkness [10] as it is radiated by the human face due to internal heat. Thermal IR captures subsurface features believed to be unique to individuals [11]. On the downside, thermal IR imagery needs to be radiometrically calibrated for each photo session as the calibration has a limited life 
span [12] i.e. the characteristics of FPA (Focal Plane Array) changes over time, no two FPAs have the same response, and the response changes with ambient conditions. Moreover, glasses are completely opaque to thermal IR 12 .

The availability of many different biometric modalities of the face raises an important question. Which modality is the best for face recognition? Currently there is no agreement on what is the correct answer to this question. While the quest for the best biometric modality of the face for recognition is likely to remain an active research area, at least for some time, many researchers have attempted to perform an unbiased comparison of some of these modalities. Socolinsky and Selinger 13 performed a comparison of thermal IR and visible image based face recognition using PCA, LDA, LFA (Local Feature Analysis) 14 and ICA (Independent Component Analysis) 15]. Their results show that all four algorithms give higher recognition performance on the thermal IR images compared to the visible light images. The database used by Socolinsky and Selinger 13 contained illumination and expression variations.

Chen et al. [11] also compared thermal IR and visible image based face recognition but on a larger database and with greater time lapse between the acquisition of training and test images compared to 13. They reported that in the case of no time-lapse in the acquisition sessions, there is negligible difference between thermal IR and visible image based recognition using PCA. In time-lapse recognition, the recognition rate of both visible and thermal IR images dropped; however PCA-based recognition performed better on visible light images.

Li et al. 9] compared the performance of visible and NIR image-based face recognition under weak illumination and reported that NIR performs better than visible images as the former produced better inter-class separation between different identities using an LBP-based (Linear Binary Patterns) [16] AdaBoost classifier. Chang et al. [17] performed a comparison of visible image and $3 \mathrm{D}$ face recognition using $\mathrm{PCA}$ and reported that both modalities give equal performance.

In almost all existing comparisons, the common criterion is the recognition rate of different face modalities using the same recognition algorithms. Such comparisons are not completely unbiased as the same recognition algorithm or features may not be suitable for every modality of the face. Moreover, an important aspect overlooked in these comparisons is the amount of variation present in each modality which will ultimately effect the maximum database size each modality can handle. To the best of our knowledge, existing literature does not compare different biometric modalities of the face using the amount of captured variation as a criterion. This paper attempts to cover these gaps and presents a comparison between the most common biometric modalities of the face using captured variation as a criterion. Experiments are performed on the Equinox 12 and the FRGC [18] databases and comparisons are presented for visible versus thermal IR and range images. Our results indicate that visible images capture more interpersonal variations compared to thermal IR and range images.

The rest of the paper is organized as follows. Section 2 gives justification of the criterion used in this paper to compared different biometric modalities of 
the face. Section 3 describes the databases used and the database normalization procedures used in our experiments. Section 4 describes the experimental setup and the results. Section 5 gives conclusions and some analysis of our results.

\section{Justification of Comparison Criterion}

The recognition performance of any biometric modality is a function of the following factors. (1) The accuracy of measurement. (2) Invariance of the biometric and the measurement process to extrinsic factors e.g. ambient conditions, temperature, illumination. (3) Invariance of the biometric to intrinsic properties e.g. facial expression, pose etc. (4) The feature extraction and classification algorithms. (5) Variance in the biometric modality. By in large, existing literature has focused on the first four factors for comparing different biometric modalities of the face. Socolinsky and Selinger [13] compared visible and thermal IR images for face recognition using different classifiers. However, they did not control illumination making their experiments a test of robustness of the modalities to illumination. Robustness of facial biometrics to extrinsic and intrinsic factors is important however, it is not the focus of this paper as these factors can be controlled in some applications e.g. when the subject is cooperative.

In this paper, we mainly focus on the last factor because we believe that variance in a biometric modality is an important indicator of its ability to perform well in large databases. As a crude example, imagine we were to identify people based on their heights or fingerprints. If we validate our classifiers using a small database of less than 20 individuals, both biometrics are likely to give similar performance. However, if we increase the database size from 20 to 2000, the fingerprints based classifier will give far better performance than the height based classifier. This is mainly because the variance in fingerprints of different individuals is more compared to their heights.

Ideally, the number of individuals enrolled using the biometric modalities under comparison must be increased to an extent that the gap between their recognition performances starts increasing. Moreover, the enrollment must be done under controlled conditions as the test is not of their robustness to extrinsic and intrinsic factors. This is a very difficult task and we argue that in the absence of such data, the variance in the biometric modalities is a good indicator of its ability to accommodate large databases. Statistically, the PDFs (Probability Distribution Functions) can be estimated from a small sample taken randomly from the population.

We give a more intuitive example using PDFs. Imagine two biometric modalities whose PDFs can be approximated by normal distributions as shown in Fig. 1. However, one biometric modality has higher variance than the other. As more and more measurements are taken, they are likely to fall within the same bins of the distribution and the more the variance in the distribution, the more will be the separation between these bins. In person identification, each bin corresponds to a unique individual and it is desirable to have as much separation between the bins as possible so that a person is correctly classified to the correct bin even in the presence of noise. 


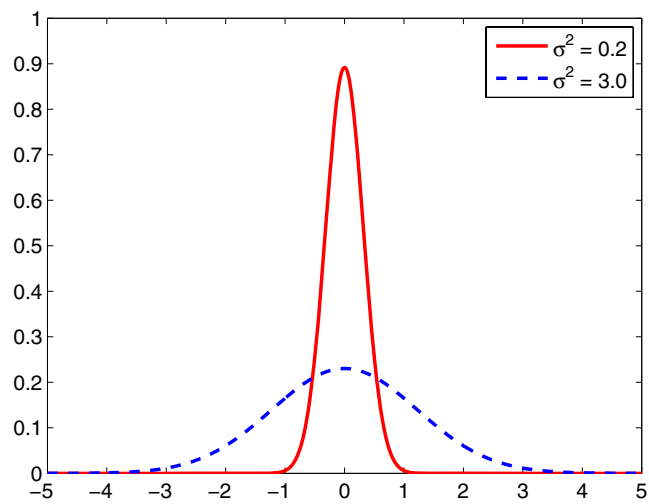

Fig. 1. Probability Distribution Functions with different variances

Variance in the biometric modalities could be measured in the raw data however, biometrics acquired through imaging have very high dimensionality and contains noise. Therefore, it is advantageous to consider only the most significant eigenvectors of the data by performing Principal Component Analysis. Recall that we are assuming controlled condition which means no intra-class variation in the data. Therefore, only a single sample per individual per modality is sufficient.

\section{Data Normalization}

We used the Equinox database [12] for comparing visible images with thermal IR images. For comparison of visible images with range images (3D data), we used the FRGC (Face Recognition Grand Challenge) database 18. While performing the comparison between two biometric modalities of the face, every possible effort was made to make all other variables constant i.e. a constant frontal illumination was chosen and coregistered images belonging to the same acquisition session were chosen. In the case of the Equinox database, the same frame number was chosen for all individuals so that every individual has the same facial expression across different modalities and there is a one-to-one correspondence between the pixels of different modalities. Note that this paper compares the amount of interpersonal variation captured by different biometric modalities of the face as opposed to the invariance of the modalities to external variations such as illumination.

The corresponding visible and thermal IR images of the Equinox database were already coregistered. However, there were some scale and pose variations between the images of different individuals. Scale and pose were normalized across all the images by manual identification of four landmarks on the visible images and transforming both the visible and thermal IR images to the same coordinates. Note that the same transformation can be used for the corresponding thermal IR and visible images as they were already coregistered. A mask 

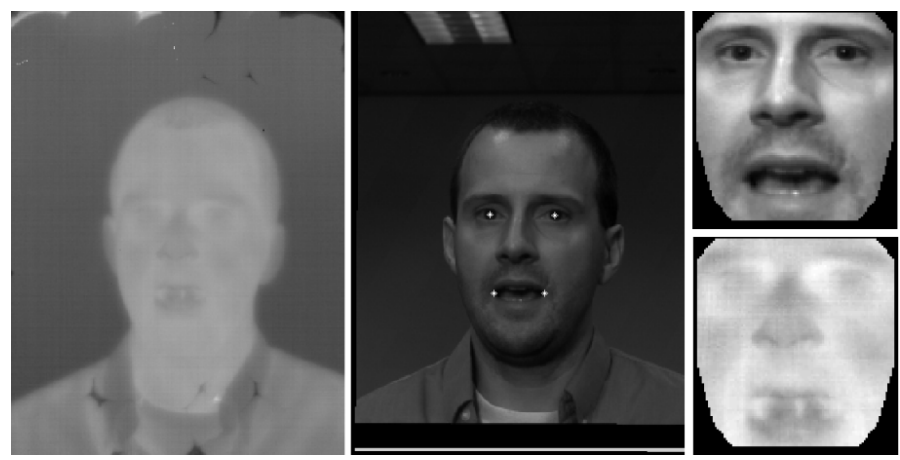

Fig. 2. Thermal IR image (left) and its coregistered visible light image (center) are normalized and cropped (right) using four landmarks selected on the visible image
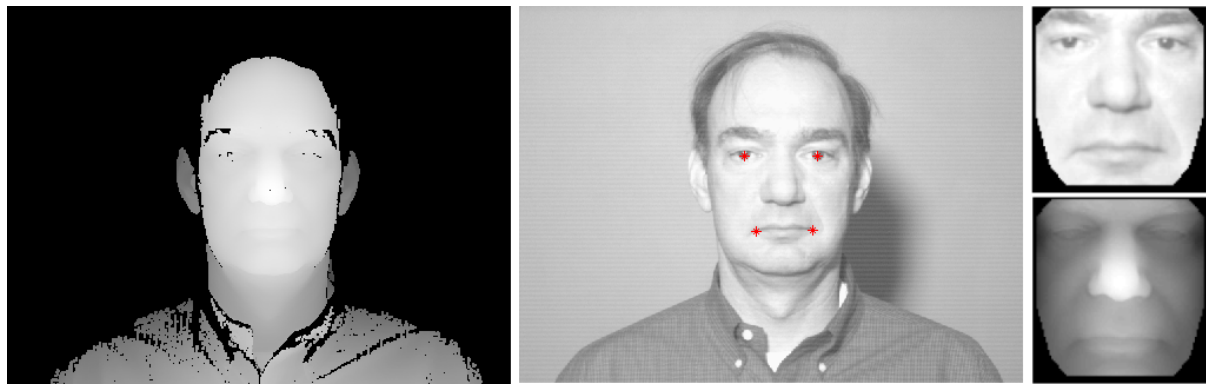

Fig. 3. Range image (left) and its coregistered visible image (center) are normalized and cropped (right) by manually identifying four landmarks. The range image is also preprocessed to remove holes and spikes.

was used to remove the background. Fig. 2 shows a sample pair of coregistered thermal IR and visible images before and after normalization.

For comparison between visible and range (3D) images, we used the FRGC database. The range images were preprocessed to remove spikes using a neighborhood distance constraint and fill holes using cubic interpolation. The visible and range image pairs where then normalized in a similar way to the thermal IR images i.e. by identifying four landmarks on the visible image and using the same transformation to normalize the visible and range images. Fig. 3 shows a sample pair of visible and range image before and after normalization.

\section{Experiments and Results}

Ideally, if a single database of coregistered images in the visible, thermal IR range and 3D data were available for a significant number of subjects, we would have performed a single experiment to compare the three modalities. However, 
there is no public database which offers simultaneously acquired and coregistered images in all the three modalities. Therefore, we performed two experiments. The first one to compare visible and thermal IR using the Equinox database and the second one to compare visible and range images using the FRGC database.

\subsection{Experiment 1}

The first experiment compares visible and thermal IR images. There were 89 subjects for which both modalities were available in the Equinox database. A single pair of visible and its coregistered thermal IR image was chosen for each subject. The images were normalized as discussed in the previous section and then projected to the PCA space. Each image in a given modality was converted to a column vector and placed in a matrix $\mathbf{I}=\left[I_{1}, I_{2}, \ldots I_{n}\right]$, where $I_{1}$ is the column vector of the first image and $n$ is the total number of images. Next, the covariance matrix of $\mathbf{I}$ is calculated.

$$
\begin{aligned}
& m=\frac{1}{n} \sum_{i=1}^{n} I_{i}, \\
& C=\frac{1}{n} \sum_{i=1}^{n} I_{i} I_{i}^{T}-m m^{T},
\end{aligned}
$$

where $m$ is the mean image and $C$ is the covariance matrix. The eigenvalues of the covariance matrix are calculated as follows:

$$
C V=D V,
$$

where $V$ is the matrix of eigenvectors and $D$ is the diagonal matrix of eigenvalues $\lambda$. The eigenvalues are sorted in decreasing order $\left(\lambda_{1}\right.$ being the highest eigenvalue) and the ratio $\psi_{k}$ of the sum of the first $k$ eigenvalues to the sum of all the eigenvalues is calculated

$$
\psi_{k}=\frac{\sum_{i=1}^{k} \lambda_{i}}{\sum_{i=1}^{n} \lambda_{i}} .
$$

The ratio $\psi_{k}$ shows the fidelity of reconstruction of the original image from its highest $k$ eigenvalues. The higher the value of $\psi_{k}$ for a given $k$, the smaller is the variation in the images. In all our experiments $\psi_{k}$ is used as a metric to compare the variation between different modalities. Higher values of $\psi_{k}$ for a given modality mean that there is less variation in the modality. Fig. 4 -a compares the $\psi_{k}$ curves of visible and thermal IR images. The figure clearly shows that visible images capture more variation in the human faces compared to thermal IR images.

\subsection{Experiment 2}

This experiment compares visible and range (3D) images. We picked a single pair of visible and range images for 89 subjects from the training set of the 


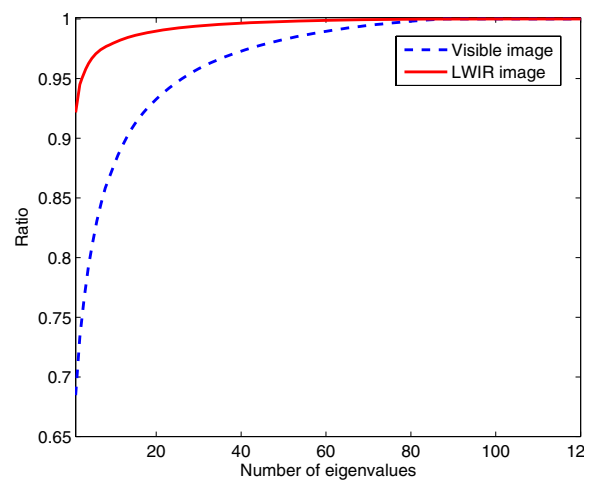

(a)

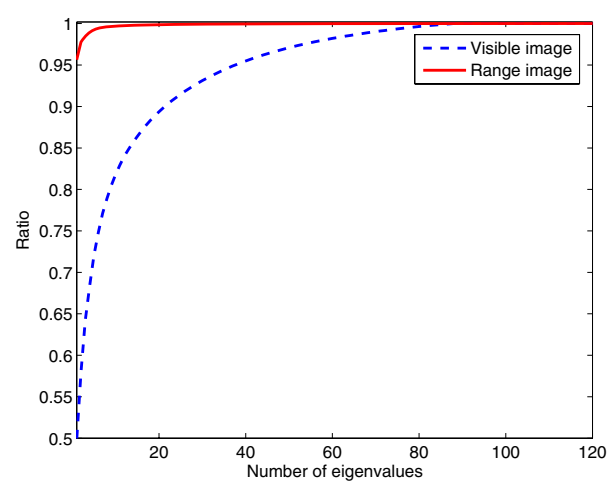

(b)

Fig. 4. The ratio $\psi_{k}$ (vertical axis) versus the number of eigenvalues $k$ (horizontal axis). (a) Results of experiment 1 . Visible images capture more interpersonal variation in human faces compared to thermal IR images. (b) Results of experiment 2. Interpersonal variation in facial range images is significantly lower than visible images.

Table 1. Number of eigenvectors required to preserve $95 \%$ variance in facial images of different modalities. Higher values correspond to greater variation in the images.

\begin{tabular}{|l|c|c|c|c|}
\hline Database & \multicolumn{2}{|c|}{ Equinox } & \multicolumn{2}{c|}{ FRGC } \\
\hline Subjects & \multicolumn{2}{|c|}{89} & Visible Image & Range Image \\
\hline Modality & Visible Image & Thermal IR Image & 38 & 1 \\
\hline Eigenvalues & 27 & 3 & 38 \\
\hline
\end{tabular}

FRGC data (Spring2003range [18]) as these images were acquired in controlled illumination with neutral facial expressions. The reason for choosing 89 images was to make the number of subjects compatible with experiment 1 . Moreover, the 89 images were hand picked to avoid image pairs with poor coregistration, excessive number of holes (missing data) in the range image and other types of corrupted imagery. Note that hand picking good images does not bias our experiment as we are not performing recognition. In fact, this ensures that the data are not biased towards the range images which would otherwise show more variation due to sensor problems i.e. holes and spikes. The visible and range images were normalized as discussed in the previous section and then projected to the PCA space as described in Section 4.1 Fig. 4 - b shows the $\psi_{k}$ curves for the two modalities. Notice that visible images capture more interpersonal variation compared to range images. In fact the difference is more significant in this case.

In Principal Component Analysis based compression techniques, the number of significant eigenvectors is usually chosen such that $95 \%$ of the total variance is preserved in the data (in our case images). Therefore, we chose $95 \%$ variance 
as a benchmark and compared the number of significant eigenvectors required by each modality to achieve this benchmark. The results of both experiments are compiled in Table 1 .

\section{Conclusion and Analysis}

This paper presented a comparison of three different biometric modalities of the face. Unlike previous studies, which used face recognition rate as a criterion, this paper used the amount of variation as a comparison criterion. This criterion is significant as it will influence the performance of a given modality with increasing database size. Practical face recognition systems are expected to operate with very large databases, which are hard to generate for the purpose of experimental analysis. Our results conclude that visible images capture more interpersonal variation in the human faces compared to thermal IR and range images.

The outcomes of our experiments are not surprising. One can intuitively perceive that visible light is likely to capture more variation in human faces because under constant illumination, pose and facial expressions, the visible image is a function of two intrinsic properties of the face. These intrinsic properties are the face albedo (facial texture) and the 3D shape of the face. Even though visible image based face recognition algorithms are sometimes referred to as $2 \mathrm{D}$ face recognition algorithms, this is not entirely true as visible images also carry shape information which is exploited in shape from shading algorithms. Compared to the visible image, the thermal IR and range image each captures only a single intrinsic property of the face namely the radiated heat and the facial geometry. Human faces have the same topological shape and there is less variation in it compared to the facial texture.

\section{Acknowledgments}

The author would like to thank Equinox Corporation for providing the thermal IR data and the FRGC organizers [18] for providing the range data. This research is supported by ARC grant DP0881813.

\section{References}

1. Turk, M., Pentland, A.: Eigenfaces for Recognition. Journal of Cognitive Neuroscience 3, 71-86 (1991)

2. Zhao, W., Chellappa, R., Phillips, P.J., Rosenfeld, A.: Face Recognition: A Literature Survey. ACM Computing Survey, 399-458 (2003)

3. Belhumeur, P., Hespanha, J., Kriegman, D.: Eigenfaces vs. Fisherfaces: Recognition Using Class Specific Linear Projection. IEEE Transactions on Pattern Analysis and Machine Intelligence 19, 711-720 (1997)

4. Srivastava, S., Gupta, M., Frigyik, B.: Bayesian Quadratic Discriminant Analysis. The Journal of Machine Learning Research 8(3), 1277-1305 (2007) 
5. Lu, J., Plataniotis, K., Venetsanopoulos, A.: Regularized discriminant analysis for the small sample size problem in face recognition. Pattern Recognition Letters 24(16), 3079-3087 (2003)

6. Bowyer, K.W., Chang, K., Flynn, P.: A Survey Of Approaches and Challenges in $3 \mathrm{D}$ and Multi-modal $3 \mathrm{D}+2 \mathrm{D}$ Face Recognition. Computer Vision and Image Understanding 101(1), 1-15 (2006)

7. Blanz, V., Vetter, T.: Face Recognition Based on Fitting a 3D Morphable Model. IEEE Transactions on Pattern Analysis and Machine Intelligence 25, 1063-1074 (2003)

8. Bronstein, A.M., Bronstein, M.M., Kimmel, R.: Three-dimensional face recognition. International Journal of Computer Vision 64(1), 5-30 (2005)

9. Li, S.Z., Chu, R., Liao, S., Zhang, L.: Illumination invariant face recognition using near-infrared images. IEEE Transactions on Pattern Analysis and Machine Intelligence 29(4), 627-639 (2007)

10. Jain, A., Bolle, R., Pankanti, S.: Biometrics: Personal Identification in Networked Society. Kluwer Academic Publishers, Dordrecht (1999)

11. Chen, X., Flynn, P., Bowyer, K.: Ir and visible light face recognition. Computer Vision and Image Understanding 99(3), 332-358 (2005)

12. Socolinsky, D., Wolff, L., Neuheisel, J., Eveland, C.: Illumination invariant face recognition using thermal infrared imagery. In: IEEE Conference on Computer Vision and Pattern Recognition, vol. 1, pp. 527-534 (2001)

13. Socolinsky, D., Selinger, A.: A comparative analysis of face recognition performance with visible and thermal infrared imagery. In: International Conference on Pattern Recognition, vol. 4, pp. 217-222 (2002)

14. Penev, P., Attick, J.: Local Feature Analysis: A general statistical theory for object representation. Network: Computation in Neural Systems 7(3), 477-500 (1996)

15. Bartlett, M.S., Lades, H.M., Sejnowski, T.: Independent Component Representation for Face Recognition. In: SPIE, pp. 528-539 (1998)

16. Ahonen, T., Hadid, A., Pietikainen, M.: Face Recognition with Local Binary Patterns. In: Pajdla, T., Matas, J(G.) (eds.) ECCV 2004. LNCS, vol. 3021, pp. 469 481. Springer, Heidelberg (2004)

17. Chang, K., Bowyer, K., Flynn, P.: Multi-Modal 2D and 3D Biometrics for Face Recognition. In: IEEE Analysis and Modeling of Faces and Gestures, pp. 187-194 (2003)

18. Phillips, P.J., Flynn, P.J., Scruggs, T., Bowyer, K.W., Chang, J., Hoffman, K., Marques, J., Min, J., Worek, W.: Overview of the Face Recognition Grand Challenge. In: IEEE Computer Vision and Pattern Recognition, pp. 947-954 (2005) 